\title{
Decreased gastrointestinal toxicity associated with a novel capecitabine schedule (7 days on and 7 days off):
} a systematic review

\author{
Karen A Cadoo ${ }^{1,2}$, Devika Gajria ${ }^{1,2}$, Emily Suh ${ }^{1}$, Sujata Patil ${ }^{3}$, Maria Theodoulou ${ }^{1,2}$, Larry Norton ${ }^{1,2}$, Clifford A Hudis $^{1,2}$ and \\ Tiffany A Traina ${ }^{1,2}$
}

\begin{abstract}
Capecitabine is widely used in the management of metastatic breast cancer; however, drug delivery is limited by gastrointestinal and other toxicity. We employed mathematical modeling to rationally design an optimized dose and schedule for capecitabine of 2,000 mg twice daily, flat dosing, 7 days on, 7 days off. Preclinical data suggested increased efficacy and tolerability with this novel dosing, and three early-phase clinical trials have suggested a favorable toxicity profile. To further define the tolerability of this regimen, we conducted a systematic review of the gastrointestinal adverse events of patients on these studies. This review demonstrated a favorable gastrointestinal toxicity profile with capecitabine in this novel schedule when given as single agent or in combination therapy with either bevacizumab or lapatinib. No patients discontinued therapy for gastrointestinal toxicity, and there were no grade 4 or 5 gastrointestinal toxicities reported. Grade 3 or greater diarrhea occurred in two (2\%); grade 2 or greater mucositis, constipation, and vomiting were reported in three (4\%) patients. We conclude that capecitabine administered on a 7 days on, 7 days off schedule has limited gastrointestinal toxicity. Our methodology was based on an analysis of individual patient toxicity data from one phase I single-agent capecitabine and two phase II capecitabine combination studies (with bevacizumab and lapatinib, respectively), focusing specifically on gastrointestinal toxicity.
\end{abstract}

npj Breast Cancer (2016) 2, 16006; doi:10.1038/npjbcancer.2016.6; published online 30 March 2016

\section{INTRODUCTION}

In spite of significant advances over the last decades in the management of metastatic breast cancer, cure remains an elusive goal. Quality of life on treatment is of major importance and underscores the need to minimize toxicity while preserving or, ideally, improving the efficacy of drugs with known anticancer activity. Models that explore optimum drug delivery are a critical component of rational clinical trial design.

Capecitabine is an orally administered fluoropyrimidine that is absorbed intact through the intestinal wall and converted via three enzymatic reactions to 5 -fluorouracil. ${ }^{2}$ The rate-limiting step in this process is the final reaction, catalyzed by thymidine phosphorylase. ${ }^{2}$ In many cancers, including breast and gastrointestinal, thymidine phosphorylase is found in higher levels in tumor than in surrounding normal tissue, allowing relatively selective tumor activity. ${ }^{2}$ Several studies have demonstrated the activity of capecitabine in patients with metastatic breast cancer, ${ }^{3-5}$ and it is widely used in the management of this and gastrointestinal malignancies. ${ }^{6-8}$

The United States Food and Drug Administration (FDA) approved capecitabine at $1,250 \mathrm{mg} / \mathrm{m}^{2}$ twice daily, with 14 days continuous dosing followed by a 7-day break (14-7). However, toxicity is a significant issue at this dose and schedule, with the most frequent adverse events being palmar-plantar erythrodysesthesia (PPE), diarrhea, and stomatitis. Diarrhea occurs in $28-54 \%$ of patients receiving capecitabine, with grade $3 / 4$ diarrhea experienced by $7-19 \%$ of patients. ${ }^{9}$ Dose reductions are required in $41-65 \%$ of patients receiving capecitabine, ${ }^{9}$ with a median time to dose reduction of $<2$ months. ${ }^{10}$ Therefore, in clinical practice an empiric schedule of $1,000 \mathrm{mg} / \mathrm{m}^{2}$ twice daily, 14 days on, 7 days off, is frequently employed to improve tolerability. ${ }^{9,10}$ Toxicity is still a significant issue; dose modification is frequently required, and the efficacy of these alternative schedules has not been prospectively determined. ${ }^{9,11}$ Of note, a phase II prospective study failed to demonstrate non-inferiority for an empirically selected lower dose capecitabine regimen. ${ }^{12}$

Norton-Simon modeling can accurately predict future behavior of tumor systems on the basis of early growth measurements. ${ }^{13}$ In addition, it has determined that the rate of tumor regression is proportional to the rate of tumor growth. ${ }^{1,14}$ This model suggests that chemotherapy delivery at increased dose density can increase cancer cell kill by minimizing the opportunity for regrowth between cycles of therapy. ${ }^{1}$ This concept has been borne out in the improved outcomes demonstrated with 'dose dense' adjuvant chemotherapy in breast cancer. ${ }^{15}$ Our group applied NortonSimon modeling to capecitabine dosing and observed that the maximum effect of capecitabine is reached at $\sim 8$ days. ${ }^{16}$ Therefore, further dosing through day 14 contributes to toxicity without enhancing efficacy. ${ }^{16}$ This provided a rationale for an alternative, optimized dosing schedule that has been rationally rather than empirically designed.

Preclinical xenograft models testing a 7 day on, 7 day off (7-7) schedule allowed for higher dosing and increased efficacy compared with conventional (14-7) scheduling. ${ }^{16}$ A phase I study

\footnotetext{
${ }^{1}$ Breast Medicine Service, Department of Medicine, Memorial Sloan Kettering Cancer Center, New York, NY, USA; ${ }^{2}$ Weill Medical College of Cornell University, New York, NY, USA and ${ }^{3}$ Department of Epidemiology and Biostatistics, Memorial Sloan Kettering Cancer Center, New York, NY, USA.

Correspondence: KA Cadoo (cadook@mskcc.org)

Received 20 January 2015; revised 10 November 2015; accepted 7 December 2015
} 


\begin{tabular}{|c|c|c|c|c|}
\hline$N=81$ & Grade 1 & Grade 2 & Grade 3 & Grade 4 \\
\hline Nausea & 40 (49\%) & $2(2 \%)$ & - & - \\
\hline Diarrhea & 39 (48\%) & $6(7 \%)$ & $2(2 \%)$ & - \\
\hline Mucositis & 27 (33\%) & $2(2 \%)$ & $1(1 \%)$ & - \\
\hline Constipation & $22(27 \%)$ & $3(4 \%)$ & - & - \\
\hline Vomiting & $14(17 \%)$ & $3(4 \%)$ & - & - \\
\hline
\end{tabular}

Maximal Grade, NCI CTCAE version 3. No Grade 5 toxicities observed.

of single-agent capecitabine established that the maximumtolerated dose of capecitabine in this novel schedule is 2,000 mg flat dose twice daily. ${ }^{17}$ Two phase II studies confirmed the feasibility of this capecitabine schedule in combination with the biologic agents lapatinib and bevacizumab for patients with metastatic HER2-positive and HER2-negative breast cancer, respectively. ${ }^{18,19}$ Despite the potentially higher daily dose administered with this regimen, capecitabine was well tolerated in the 7-7 schedule. In particular, we observed improved gastrointestinal tolerability with $>$ grade 2 diarrhea of just $5-26 \%$ reported on the individual studies of the 7-7 schedule. ${ }^{17-19}$ To further define this tolerability, we conducted a systematic review of the gastrointestinal toxicity observed in patients on these early-phase studies that explored the capecitabine (7-7) dosing schedule.

\section{RESULTS}

Patient population

This analysis includes 81 patients evaluable for toxicity; 18 received single-agent capecitabine, 40 received capecitabine and bevacizumab, and 23 received capecitabine and lapatinib. The median age of patients was 52 years (range, 29-73 years) and the median ECOG performance status was 0 . Sixty-two patients (76\%) had visceral tumor involvement. The median number of prior chemotherapies for metastatic breast cancer was $0(0-2)$.

\section{Therapy delivery}

The median number of treatment cycles delivered was 6 (range, $1-40)$. Seventeen patients $(21 \%)$ received $\geqslant 12$ months of therapy. No patients discontinued therapy for gastrointestinal toxicity. Treatment discontinuation was due to progressive disease in 54 patients $(67 \%)$, toxicity in $15(19 \%)$, withdrawal of consent in 4 $(5 \%)$, and other reasons in $8(10 \%)$. Of the patients who discontinued therapy for toxicity, one was receiving single-agent capecitabine, 10 were receiving capecitabine with bevacizumab, and 4 were receiving capecitabine with lapatinib. Treatment discontinuations for toxicity deemed possibly related to capecitabine included palmar-plantar erythrodysesthesia in six patients and laboratory abnormalities in four. Other reasons for patients to discontinue therapy for toxicity included atrial fibrillation, pulmonary embolus, and headache attributed to bevacizumab; rash attributed to lapatinib; and gout. Dose reduction or delay due to gastrointestinal toxicity occurred in five $(6 \%)$ and four $(5 \%)$ patients, respectively.

\section{Gastrointestinal toxicities}

These regimens were well tolerated with minimal gastrointestinal toxicity. Table 1 lists the treatment-related gastrointestinal toxicities of all grades. There were no grade 4 or 5 gastrointestinal toxicities reported. Grade 3 or greater diarrhea occurred in two (2\%) patients; grade 2 or greater mucositis, constipation, and vomiting were reported in three (4\%) patients.
Palmar plantar erythrodysesthesia (PPE)

There were no grade 4 or 5 PPE events. Grade 3 PPE occurred in 12 (15\%) and grade 2 in 32 (40\%) patients.

\section{DISCUSSION}

Capecitabine is widely used in the management of metastatic breast and gastrointestinal cancers. Given the palliative goal of therapy for advanced disease in these malignancies, therapeutic tolerance of active agents is of critical importance. Unfortunately, gastrointestinal toxicity is a limiting issue with this drug at the conventional dose and schedule. ${ }^{9}$

Despite the widespread use of this drug, the optimal capecitabine dose for patients with metastatic breast cancer has not been defined. ${ }^{20}$ A number of empirically designed capecitabine dosing schedules have been employed in an effort to increase tolerance and facilitate continued drug delivery. ${ }^{9,12}$ However, the efficacy of these regimens has not been prospectively demonstrated, ${ }^{9,11}$ and it is not clear that this empiric dosing has validity. In fact, as noted above, a randomized phase II study failed to demonstrate the non-inferiority of lower dose capecitabine in combination with docetaxel. ${ }^{12}$ In addition, a two-stage study exploring the feasibility of fixed-dose single-agent capecitabine $3,000 \mathrm{mg}$ per day, in divided doses, failed to meet its first-stage response end point and was closed. ${ }^{21}$ We employed NortonSimon mathematical modeling to rationally design a novel and optimal dose and schedule of capecitabine. ${ }^{16}$ Modeling suggested an optimized regimen of capecitabine twice daily 7-7. In preclinical models, this improved survival over conventional scheduling. Early-phase clinical studies have established the recommended dose and have demonstrated the feasibility of this approach. ${ }^{16-19}$ Data pertaining to the efficacy of this approach is outstanding and is the subject of an ongoing randomized phase III study led by the Latin American and Caribbean Society of Medical Oncology (SLACOM). This trial is comparing conventional capecitabine scheduling with the 7-7 schedule (NCT02028494) and is powered to detect a progression-free survival benefit with the novel regimen.

In this systematic review, we present individual patient gastrointestinal toxicity from one phase I study of single-agent capecitabine and two phase II studies combined with the biologic agents bevacizumab and lapatinib. ${ }^{17-19}$ These data represent a heterogenous patient population receiving these three therapeutic regimens, each with the novel capecitabine backbone schedule as described. There were no grade 4 gastrointestinal toxicities observed with rare occurrences of grade 3 diarrhea and constipation. In addition, the rates of grade $\geqslant 2$ gastrointestinal toxicity were minimal, with only diarrhea reaching above $5 \%$ incidence. This appears to compare favorably with historical data from previous randomized studies of capecitabine with bevacizumab and lapatinib 22,23 (Table 2). Miller et al. noted $\geqslant$ grade 2 diarrhea in $26 \%$ and $28 \%$ of patients receiving capecitabine alone and capecitabine with bevacizumab, respectively. ${ }^{22}$ Similarly, Geyer et al. reported $\geqslant$ grade 2 diarrhea in $25 \%$ and $33 \%$ of patients receiving capecitabine versus capecitabine and lapatinib, respectively. ${ }^{23}$ In that study, $140(65 \%)$ of patients in the capecitabine group and 181 (79\%) of patients in the combination group required a dose reduction for toxicity; however, the toxicities necessitating these reductions were not specified. Notably, no patients in the current systematic review came off study for gastrointestinal toxicity, and 17 patients (21\%) were on this cytotoxic therapy for over a year.

Hand-foot syndrome, ${ }^{9}$ or PPE, also significantly limits capecitabine delivery. Exploration of this symptom was not the primary end point of this review; however, given the significance of this toxicity clinically, it has also been highlighted. Six of the patients who discontinued therapy for toxicity that was possibly related to 
capecitabine did so for PPE. There was no grade 4 PPE, and the rates of grade 2 and 3 PPE ( $40 \%$ and 15\%, respectively) are similar to prior data-Miller et al. ${ }^{22}$ reported $36-42 \%$ grade 2 and $24-28 \%$ grade 3 PPE, while Geyer et al. ${ }^{23}$ reported $26-32 \%$ grade 2 and $7-11 \%$ grade 3 PPE.

While the efficacy of this approach has to be determined, the improved gastrointestinal tolerability of this novel regimen does not appear to be at the cost of disease control. The median progression-free survival of the phase I study was not reported; however, in the phase II studies it was 8.0 and 9.4 months when combined with bevacizumab and lapatinib, respectively. ${ }^{18,19}$ Although it is not possible to make cross-trial comparisons, this is reasonable in the context of previous data. The median progression-free survival of the phase III studies of capecitabine with bevacizumab and lapatinib was 4.87 months $^{22}$ and 8.4 months, ${ }^{23}$ respectively.

Acknowledging the limitations of this retrospective systematic review of prospectively collected data, our study provides an encouraging signal about the tolerability of this novel dose and schedule. The favorable gastrointestinal toxicity profile demonstrated may facilitate prolonged drug delivery with reasonable quality of life. We propose that this novel dose and schedule of capecitabine optimizes the therapeutic index. As noted, the efficacy of this novel approach is the subject of an ongoing phase III clinical trial.

The potential for this dose and schedule to decrease toxicity and increase drug compliance has wide-reaching public health implications given the broad use of this drug worldwide in breast and gastrointestinal cancers. In addition, it has the potential to reduce drug cost: in this novel schedule, patients receive capecitabine for 14 days out of every 28 , as opposed to 14 out of every 21 in conventional scheduling.

Mathematical modeling employed in the design of this regimen allows for dose and schedule determination based on efficacy by defining the point of maximum tumor perturbation. ${ }^{16}$ This moves away from the traditional drug development focus of maximumtolerated toxicity rather than therapeutic index. The proposed novel capecitabine regimen also moves away from conventional dosing based on body surface area, a widely used practice in drug development that has not been well validated. ${ }^{24}$ This regimen has significant implications for future drug development and will potentially provide proof of principal that rational dose and schedule design, determined by mathematical models, may efficiently optimize activity and reduce toxicity.

We have demonstrated that this novel dose and schedule of capecitabine causes minimal and tolerable gastrointestinal toxicity. We eagerly await the outcome of the ongoing phase III study that will determine the relative efficacy of this approach.

\section{MATERIALS AND METHODS}

In reaching our conclusions from this review, our methodology, based on an analysis of individual patient toxicity data from one phase I single-agent capecitabine and two phase II capecitabine combination studies (with bevacizumab and lapatinib, respectively), focused specifically on gastrointestinal toxicity.

This analysis combines the primary toxicity data from three prospective single-institution studies exploring the feasibility of a novel capecitabine dosing schedule as previously described. ${ }^{17-19}$ These studies included patients with metastatic breast cancer and measurable disease per RECIST criteria. Patients with ECOG performance status $\leqslant 2$ and adequate hematologic, hepatic, and renal function were eligible. Each treatment cycle was 28 days, and disease evaluation occurred every 12 weeks with scans assessed by RECIST. For selected eligibility criteria and the design of each study, see the study characteristics table (Table 3). All studies were approved by the Memorial Sloan Kettering Cancer Center Institutional Review Board, and patients were provided written informed consent. Individual patient toxicity data from these studies were prospectively collected and graded using NCI CTCAE version 3 terminology, and 
Table 3. Individual study characteristics

\begin{tabular}{|c|c|c|c|}
\hline & Study $A^{17}$ & Study $B^{19}$ & Study $C^{18}$ \\
\hline Study phase & Phase I & Phase II & Phase II \\
\hline Therapy & $\begin{array}{l}\text { Capecitabine } 2,000 \mathrm{mg} \text { orally twice } \\
\text { daily, } 7 \text { days on } 7 \text { days off }\end{array}$ & $\begin{array}{l}\text { Capecitabine } 2,000 \mathrm{mg} \text { orally twice } \\
\text { daily, } 7 \text { days on } 7 \text { days off } \\
\text { Bevacizumab } 10 \mathrm{mg} / \mathrm{kg} \text { intravenously } \\
\text { every } 2 \text { weeks }\end{array}$ & $\begin{array}{l}\text { Capecitabine } 2,000 \mathrm{mg} \text { orally twice } \\
\text { daily, } 7 \text { days on } 7 \text { days off } \\
\text { Lapatinib } 1250 \mathrm{mg} \text { orally daily }\end{array}$ \\
\hline $\begin{array}{l}\text { Selected patient } \\
\text { eligibility criteria }\end{array}$ & $\begin{array}{l}\text { Any number of prior therapies } \\
\text { No prior fluoropyrimidine therapy for } \\
\text { MBC or as adjuvant therapy within } \\
6 \text { months of enrollment } \\
\text { Patients with HER2+ disease were } \\
\text { eligible if they were not candidates for } \\
\text { trastuzumab therapy }\end{array}$ & $\begin{array}{l}\text { Any number of prior therapies } \\
\text { No prior fluoropyrimidine therapy for } \\
\text { MBC } \\
\text { Patients with HER2+ disease were } \\
\text { eligible if they were not candidates for } \\
\text { trastuzumab therapy }\end{array}$ & $\begin{array}{l}\text { No more than two prior } \\
\text { chemotherapy regimens } \\
\text { No prior fluoropyrimidine therapy for } \\
\text { MBC } \\
\text { HER } 2+\text { breast cancer with disease } \\
\text { progression on prior trastuzumab } \\
\text { therapy }\end{array}$ \\
\hline Study design & $3+3$ Dose escalation & Non-randomized open label phase II & Non-randomized open label phase II \\
\hline Primary end point & MTD of capecitabine in $7 / 7$ schedule & $\begin{array}{l}\text { Overall response rate for the } \\
\text { combination }\end{array}$ & $\begin{array}{l}\text { Overall response rate for the } \\
\text { combination }\end{array}$ \\
\hline Safety assessments & $\begin{array}{l}\text { Patients were evaluated for toxicity } \\
\text { weekly during cycle } 1 \text { and every } \\
2 \text { weeks during subsequent cycles } \\
\text { Adverse events graded using NCI CTC } \\
\text { version } 3.0 \text { (Rockville, MD, USA) }\end{array}$ & $\begin{array}{l}\text { Patients were evaluated for toxicity } \\
\text { weekly during cycle } 1 \text { and every } \\
2 \text { weeks during subsequent cycles } \\
\text { Adverse events graded using NCI CTC } \\
\text { version } 3.0\end{array}$ & $\begin{array}{l}\text { Patients were evaluated for toxicity } \\
\text { every } 2 \text { weeks during cycle } 1 \text { and } \\
\text { once per cycle thereafter } \\
\text { Adverse events graded using } \mathrm{NCl} \\
\text { CTC version } 3.0 \\
\text { LVEF was monitored by MUGA scan } \\
\text { every } 12 \text { weeks. }\end{array}$ \\
\hline
\end{tabular}

Abbreviations: LVEF, left ventricular ejection fraction; MBC, metastatic breast cancer; MTD, maximum-tolerated dose; MUGA, multigated acquisition.

attribution assigned. These data were retrospectively extracted by two independent reviewers for the purposes of this systematic review. The primary end point of interest for this review is the incidence and severity of individual gastrointestinal toxicities (nausea, diarrhea, mucositis, constipation, vomiting) with this novel capecitabine schedule. The maximum grade per patient is used as a summary measure. Given the potential for PPE to limit therapy with capecitabine, the incidence and severity of this toxicity has also been highlighted.

\section{ACKNOWLEDGMENTS}

We thank the patients, and their families, who participated in the original studies included in this systematic review. This study was funded in part through $\mathrm{NIH} / \mathrm{NCl}$ Cancer Center Support Grant P30 CA008748. We previously designed an optimized dose and schedule for capecitabine of 2,000 mg twice daily, flat dosing, 7 days on, 7 days off. Our systematic review of three early-phase studies utilizing this novel dose and schedule has demonstrated minimal and tolerable gastrointestinal toxicity.

\section{CONTRIBUTIONS}

K.A.C.*-literature search, tables, study design, data collection, data analysis, data interpretation, writing. D.G.- - literature search, tables, study design, data collection, data analysis, data interpretation, writing. E.S.- - literature search, tables, study design, data collection, data analysis, data interpretation, writing. S.P.—study design, data collection, data analysis, data interpretation, writing. M.T.- data collection, data analysis, data interpretation. L.N.-study design, data analysis, data interpretation, writing. C.H.-study design, data analysis, data interpretation, writing. T.T.-literature search, tables, study design, data collection, data analysis, data interpretation, writing. *Guarantor

\section{COMPETING INTERESTS}

The authors declare no conflict of interest.

\section{REFERENCES}

1. Norton, L. Conceptual and practical implications of breast tissue geometry: toward a more effective, less toxic therapy. Oncologist 10, 370-381 (2005).

2. Diasio, R. B. An evolving role for oral fluoropyrimidine drugs. J. Clin. Oncol. 20, 894-896 (2002).
3. Reichardt, P. et al. Multicenter phase II study of oral capecitabine (Xeloda") in patients with metastatic breast cancer relapsing after treatment with a taxanecontaining therapy. Ann. Oncol. 14, 1227-1233 (2003).

4. Fumoleau, P. et al. Multicentre, phase II study evaluating capecitabine monotherapy in patients with anthracycline- and taxane-pretreated metastatic breast cancer. Eur. J. Cancer 40, 536-542 (2004).

5. O'Shaughnessy, J. A. et al. Randomized, open-label, phase II trial of oral capecitabine (Xeloda) versus a reference arm of intravenous CMF (cyclophosphamide, methotrexate and 5-fluorouracil) as first-line therapy for advanced/metastatic breast cancer. Ann. Oncol. 12, 1247-1254 (2001).

6. Hoff, P. M. et al. Comparison of oral capecitabine versus intravenous fluorouracil plus leucovorin as first-line treatment in 605 patients with metastatic colorectal cancer: results of a randomized phase III study. J. Clin. Oncol. 19, 2282-2292 (2001).

7. Van Cutsem, E. et al. Oral capecitabine compared with intravenous fluorouracil plus leucovorin in patients with metastatic colorectal cancer: results of a large phase III study. J. Clin. Oncol. 19, 4097-4106 (2001).

8. Cunningham, D. et al. Capecitabine and oxaliplatin for advanced esophagogastric cancer. N. Engl. J. Med. 358, 36-46 (2008).

9. Hennessy, B. T., Gauthier, A. M., Michaud, L. B., Hortobagyi, G. \& Valero, V. Lower dose capecitabine has a more favorable therapeutic index in metastatic breast cancer: retrospective analysis of patients treated at M. D. Anderson Cancer Center and a review of capecitabine toxicity in the literature. Ann. Oncol. 16, 1289-1296 (2005).

10. Zielinski, C., Gralow, J. \& Martin, M. Optimising the dose of capecitabine in metastatic breast cancer: confused, clarified or confirmed? Ann. Oncol. 21, 2145-2152 (2010).

11. Leonard, R., Hennessy, B. T., Blum, J. L. \& O'Shaughnessy, J. Dose-adjusting capecitabine minimizes adverse effects while maintaining efficacy: a retrospective review of capecitabine for metastatic breast cancer. Clin. Breast. Cancer. 11, 349-356 (2011).

12. Buzdar, A. U. et al. Randomized phase II non-inferiority study (NO16853) of two different doses of capecitabine in combination with docetaxel ;for locally advanced/metastatic breast cancer. Ann. Oncol. 23, 589-597 (2012).

13. Norton, L., Simon, R., Brereton, H. D. \& Bogden, A. E. Predicting the course of Gompertzian growth. Nature 264, 542-545 (1976).

14. Norton, L. \& Simon, R. The Norton-Simon hypothesis revisited. Cancer Treat. Rep. 70, 163-169 (1986).

15. Citron, M. L. et al. Randomized trial of dose-dense versus conventionally scheduled and sequential versus concurrent combination chemotherapy as postoperative adjuvant treatment of node-positive primary breast cancer: first report of Intergroup Trial C9741/Cancer and Leukemia Group B Trial 9741. J. Clin. Oncol. 21, 1431-1439 (2003). 
16. Traina, T. A. et al. Optimizing chemotherapy dose and schedule by Norton-Simon mathematical modeling. Breast Dis. 31, 7-18 (2010).

17. Traina, T. A. et al. Phase I study of a novel capecitabine schedule based on the Norton-Simon mathematical model in patients with metastatic breast cancer. J. Clin. Oncol. 26, 1797-1802 (2008).

18. Gajria, D. et al. Phase II trial of a novel capecitabine dosing schedule in combination with lapatinib for the treatment of patients with HER2-positive metastatic breast cancer. Breast Cancer Res. Treat. 131, 111-116 (2012).

19. Gajria, D. et al. Phase 2 trial of a novel capecitabine dosing schedule in combination with bevacizumab for patients with metastatic breast cancer. Cancer 117, 4125-4131 (2011).

20. Hudis, C., Traina, T. \& Norton, L. Capecitabine dosing is not yet optimized for breast cancer. Ann. Oncol. 21, 2291, author reply 2291-2292 (2010).

21. Rudek, M. A. et al. Fixed-dose capecitabine is feasible: results from a pharmacokinetic and pharmacogenetic study in metastatic breast cancer. Breast Cancer Res. Treat. 139, 135-143 (2013).
22. Miller, K. D. et al. Randomized phase III trial of capecitabine compared with bevacizumab plus capecitabine in patients with previously treated metastatic breast cancer. J. Clin. Oncol. 23, 792-799 (2005).

23. Geyer, C. E. et al. Lapatinib plus capecitabine for HER2-positive advanced breast cancer. N. Engl. J. Med. 355, 2733-2743 (2006).

24. Mathijssen R. H. et al. Flat-fixed dosing versus body surface area based dosing of anticancer drugs in adults: does it make a difference? Oncologist 12: 913-923. (2007). International License. The images or other third party material in this article are included in the article's Creative Commons license, unless indicated otherwise in the credit line; if the material is not included under the Creative Commons license, users will need to obtain permission from the license holder to reproduce the material. To view a copy of this license, visit http://creativecommons.org/licenses/ by/4.0/ 